\title{
What is the effectiveness of using conspecific or heterospecific acoustic playbacks for the attraction of animals for wildlife management? A systematic review protocol
}

Breanna J. Putman ${ }^{1,2^{*}}$ (D) and Daniel T. Blumstein ${ }^{1}$

\begin{abstract}
Background: Many animals are attracted to the sounds of their conspecifics and some are attracted to the sounds of other species. Therefore, wildlife managers have begun to capitalize on this attraction to lure animals to specific places by broadcasting vocalizations - a method referred to as acoustic playback. Playbacks have been conducted to attract animals for capture, encourage birds to use specific nest boxes, attract animals to safe locations where they can breed, or to lure animals to habitats away from human disturbances. However, there has been no evaluation of the general effectiveness of this intervention on the attraction of animals for wild life management. We describe a protocol to systematically review the literature of the effectiveness of using playbacks so that conservation practitioners can make informed wildlife management decisions.
\end{abstract}

Methods: The review will examine primary field studies that use acoustic playbacks to attract animals for wildlife management. Playbacks could be of conspecifics, heterospecifics, or both. We will search various bibliographic databases, online search engines, and specialist websites for relevant studies. We will screen studies first on title and abstract, then on full text. We will only include studies that measure quantitative results. Relevant outcomes will include the attraction of animals to playbacks and encompass various management goals: to capture the animal, to persuade the animal to use a new breeding location, and/or to persuade the animal to settle in a location during the study duration. Studies must be based on comparison between conditions before and after intervention, on comparison between treatment and control plots, or both. Inter-reviewer consistency in article inclusion will be performed with $10 \%$ of the titles and abstracts and $10 \%$ of the full texts. We will critically appraise the literature based on study type and design, sample sizes, study duration, and other aspects of methodology. We will extract data and metadata including various potential effect modifiers (e.g., taxon). Finally, we will evaluate the effectiveness of playbacks through a narrative synthesis of the evidence, and quantitative synthesis (i.e., meta-analysis) if sufficient data (more than two studies) of similar outcomes are found.

Keywords: Wildlife conservation, Conspecific attraction, Heterospecific attraction, Settlement, Breeding, Establishment, Migration, Dispersal, Broadcast, Evidence synthesis

\footnotetext{
*Correspondence: putman.bree@gmail.com

${ }^{1}$ Department of Ecology and Evolutionary Biology, University

of California, 621 Young Drive South, Los Angeles, CA 90095-1606, USA

Full list of author information is available at the end of the article
} 


\section{Background}

The phenomenon in which animals seek out and benefit from associations with conspecifics is often referred to as conspecific attraction [1]. Many species use vocalizations to communicate with and locate members of their own species. From an individual's perspective, vocalizations may be used as cues to indicate the presence and location of conspecifics, bringing animals closer and/or encouraging them to settle in areas with vocalizations; thus, behavioral decisions are frequently influenced by acoustically-transmitted social information. Conspecific attraction occurs in a variety of taxa. American toads (Anaxyrus americanus) and Cope's gray tree frogs (Hyla chrysoscellis) locate breeding aggregations via frog choruses [2], grasshopper sparrows (Ammodramus savannarum) are more likely to settle in habitats with conspecific vocalizations than without [3], and blackcapped vireos (Vireo atricapilla) are more likely to settle in nest boxes from which vireo vocalizations are broadcast compared to control nest boxes with no vocalizations [4].

In addition, the sounds of heterospecifics can influence animal behavior. Song sparrows (Melospiza melodia) living at nests where predator vocalizations were broadcast increased their antipredator behavior, brought less food to their young, and ultimately produced $40 \%$ fewer offspring compared to those living at nests where vocalizations from non-predators were broadcast [5]. Importantly, nest predation itself was eliminated by protecting their nests with chicken wire. Thus, predator's sounds alone influenced population dynamics. In terms of habitat selection, migrating newts may use the sounds of frog calls to orient toward breeding sites [6], and playbacks of heterospecific bird vocalizations can positively influence selection of nesting habitats [7].

Broadcasting recordings of animal vocalizations, called acoustic playbacks, is a common method in studies of animal behavior, particularly in communication (e.g., to study signal form, function, and evolution), in social ecology (e.g., to study social relationships) [8], and as mentioned above, in habitat selection (e.g., to study cues used to select breeding grounds). Because behavior is the key pathway through which animals interact with their environment, conservation practitioners have begun to ask whether and how sounds could be used to manage wildlife $[9,10]$. The animal behavior literature illustrates an attractive function of conspecific and heterospecific sounds (although in some cases, as in predator vocalizations, sounds may act as repellents [11]), and acoustic playbacks might offer a promising method to encourage animals to settle in desired locations; for instance, away from humans and/or in newly created or restored natural habitats.
One of the first known instances of using acoustic playbacks for wildlife management was for re-establishing an arctic tern (Sterna paradisaea) colony in Maine that had been negatively impacted by gulls [12]. Through gull control, artificial tern decoys and playbacks of nonaggressive tern vocalizations, nesting by terns at the colony was nearly doubled [12]. Since then, many studies have been conducted (e.g., $[13,14])$ to encourage wildlife habitat selection via conspecific and/or heterospecific acoustic playbacks. However, although acoustic playbacks for wildlife management are supported by theory, it is an open question as to how effective this method is, and under what circumstances (e.g., for temporary breeding or long-term establishment). For instance, playbacks do not always work to attract the target species [10]. Furthermore, we need to evaluate the influence of additional factors on its effectiveness. Some studies suggest that it is important to include heterospecific vocalizations with the conspecific vocalizations of the target species [4], and that broadcasting playbacks at night is essential for attracting migratory birds [15]. In addition, the technology used (e.g., number of speakers) and sound properties of the recordings (e.g., amplitude) could impact the effectiveness of this intervention $[8,10]$.

Human-induced environmental change will continue to impact wildlife worldwide through urban development, habitat fragmentation, pollution, and introduction of nonnative species $[16,17]$. Thus, wildlife conservation management interventions that involve the movements of animals away from these human-caused disturbances may become more important. Yet, we need to understand their effectiveness to make informed management decisions and our systematic review attempts to evaluate the effectiveness of one promising intervention.

\section{Stakeholder engagement}

This systematic review topic was proposed during a Collaboration for Environmental Evidence (CEE) training workshop (Oct 2017) aimed at increasing the use of systematic reviews for behavioral interventions in wildlife conservation management. Our advisory group consisted of 15 researchers (including the authors) from various international institutions (8 different countries) with expertise in behavioral ecology, animal cognition, conservation biology, and conservation planning. We acknowledged that conservation management decisions are often made without evidence of the effectiveness of interventions on animal behavior (see [18]). We jointly conceived of topics that are of broad importance, for which studies exist (in both primary and gray literature), and for which outcomes are not well understood (i.e., there is no general agreement upon the effectiveness of the intervention). In all, we formulated a number of systematic review 
and systematic map questions that fell within the one of three broad categories of conservation concern (see [19]): (1) using animal behavior to improve the outcomes of conservation translocations, (2) detrimental effects of noise and light pollution on wildlife populations, and (3) using behavioral cues to attract animals for conservation purposes (see Additional material in [18]).

Additional stakeholders were consulted prior to drafting this protocol. We specifically contacted wildlife conservation managers, who represent stakeholders that would use the information garnered from our review when deciding on management actions (e.g., whether and how to use playbacks to attract animals). We emailed contacts at the San Diego Zoo Global and the National Wildlife Research Center (NWRC), which is the research arm of the USDA-APHIS Wildlife Services program in the United States. We asked recipients to pass along our email to colleagues and to respond to the following questions:

- What general information would they find valuable from this systematic review?

- What sources of heterogeneity (effect modifiers) are they interested in or think will be important?

- Do they know of important benchmark studies that should be included when defining our search terms?

- Do they know particular sources of grey literature for our review topic?

- What outcome(s) (measured dependent variables) are they most interested in?

We received encouraging feedback from five conservation managers. Most (4/5) of these stakeholders were part of NWRC and worked on birds as a taxonomic group, although one had extensive experience with African wildlife conservation. Importantly, stakeholders agreed that there is a very poor understanding of the intervention, especially given that playbacks are used to both attract and repel animals. Thus, playbacks are used for a variety of management goals.

The outcomes that stakeholders brought up included not only attracting animals to a certain location for settlement or breeding, but also for capture as this can be important for many conservation programs. For instance, one stakeholder stated that managers use playback calls of lions eating to attract lions to a carcass, and acoustic playbacks to catch Northern Saw-whet Owls (Aegolius acadicus). Stakeholders were also interested in how aspects of the playbacks themselves affect outcomes. For instance, does the amplitude, frequency range, periodicity, and volume of the playback(s) matter? They also asked whether the addition of another stimulus (i.e., a visual cue) increases the effectiveness of the intervention. They were mostly interested in how the effectiveness of playbacks varies by species or guild.

\section{Objective of the review}

This systematic review aims to review the literature of the effectiveness of using playbacks for conservation, specifically aimed at understanding the utility of playbacks to attract animals to a particular location to achieve a wildlife management goal. There is one review of this topic that is specifically focused on the conservation of migratory birds [10]. We are unaware of any previous systematic reviews on this topic. Thus, our protocol illustrates the process we will follow to conduct a systematic review that follows the guidelines of the Collaboration for Environmental Evidence [20].

Using acoustic playbacks for wildlife management is not new, but no formal synthesis of their effectiveness exists to inform managers on if and when playbacks are likely to work. The systematic approach is designed to avoid bias and permit quantitative evaluation of data from previous studies that have tested this type of intervention. Hence, a systematic review should provide an objective basis for deciding if and when playbacks can be used as a tool for the management of animals.

Our primary question is related to the overall effectiveness of playbacks in attracting animals of conservation concern (Table 1). Conservation concern merely refers to the fact that the animal is the focus of wildlife management decisions (often aimed at conservation) and does necessarily imply that it is threatened or endangered. This attraction may be for several reasons including to

Table 1 Primary question components

\begin{tabular}{ll}
\hline Component & Definition \\
\hline $\begin{array}{l}\text { Population } \\
\text { Intervention }\end{array}$ & Wild animals of conservation concern \\
Comparator & No intervention in time, space, or both \\
Outcomes & $\begin{array}{l}\text { Attraction of the animals to a desired location or habitat. This could be long-term or short-term settlement depending on wildlife } \\
\text { management goals: attraction of animal for capture, occupation at desired location during the study attempt, establishment of nests } \\
\end{array}$ \\
& or burrows, establishment of territories, and/or exhibition of breeding behaviours (e.g., use of nest boxes)
\end{tabular}


capture the animal, to lure the animal to a new breeding ground, to move the animal away from humans (i.e., reduce human-wildlife conflict), and/or to persuade an animal that is dispersing to choose a particular settlement location. Species counts, although not a direct outcome, may be used to quantify one or more of the above outcomes. The intervention is deemed effective if the outcome of the study was positive. The scope will not be limited by taxon or geography as we are interested in the influence of these factors. We acknowledge that heterogeneity may come from many factors, and we list two that will be easily extracted from studies and were suggested to us by stakeholders. These secondary questions are related to how taxon and type of outcome influence the successful attraction of animals.

\section{Primary question}

What is the effectiveness of using conspecific and/or heterospecific acoustic playbacks for the attraction of animals for wildlife management?

\section{Secondary questions}

- Is the effectiveness of playbacks influenced by the type of outcome (see list in Table 1)?

- To what extent is the effectiveness of playbacks influenced by taxonomic group or guild?

\section{Methods}

\section{Searching for articles Search strategy}

We intend to search a wide range of bibliographic databases, online search engines, and gray literature sources to be as comprehensive as possible. We will not restrict searches based on date ranges or document type. Both authors are at the University of California, Los Angeles and so our searches will be limited to the subscriptions of this institution. In addition, searches will be conducted solely in English since both authors are not proficient in other languages. We will conduct web-based searches (e.g., online search engines and organizational websites) within the Google Chrome web browser using the 'incognito' mode, which keeps the browser history private and allows a person to search the web without storing local data. We will also manually clear cookies and the browser history prior to running web-based searches. Thus, previous search histories and computer location should not influence search results.

\section{Search string}

We used stakeholder engagement and conducted a scoping exercise to test alternative search strings. Stakeholders identified the outcome of capture, which was added as a search term. We used a benchmark list that included four studies (see Additional file 1) that we and stakeholders identified as well-known within the field of acoustic playbacks for conservation. These studies meet our inclusion criteria (see below) and should emerge in searches. If all four studies were not retrieved during a search, we determined that our search string terms needed to be updated. Through this scoping exercise, we determined search terms that are sensitive enough to return a broad number of studies, while also being specific enough to return relevant material (including all of our benchmark studies) (Table 2). As examples of the breadth of literature retrieved, our scoping exercise resulted in 3829 results within Web of Science and 3808 hits in ProQuest as of 8 November 2018 (see databases below).

From Table 2, the terms within each of the categories 'Population, 'Intervention', 'Outcome', 'Additional Descriptors for Intervention', and 'Additional Descriptors for Outcome' will be combined using the Boolean operator 'OR'. The five categories will then be combined using the Boolean operator 'AND'. An asterisk (*) is a 'wildcard' that represents any group of characters, including no character. Quotation marks ("') are used to search exact phrases (including hyphenated variations).

Table 2 Search terms to be used in the review (separated into the question components)

\begin{tabular}{|c|c|}
\hline Component & Search terms \\
\hline Population & 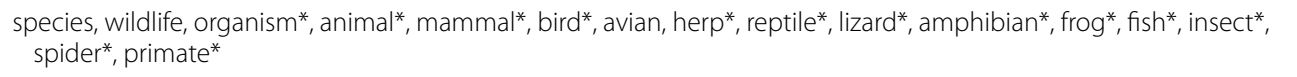 \\
\hline Intervention & $\begin{array}{l}\text { playback*, song**, call*, sound*, }{ }^{*} \text { acoustic* }{ }^{*} \text {, auditory, vocal*, broadcast* }{ }^{*} \text { comminicat* }{ }^{*} \text { tap* }{ }^{*} \text { drum* } \text { stridulate* }^{*} \text {, vibrat*, } \\
\text { decoy* }\end{array}$ \\
\hline Outcome & $\begin{array}{l}\text { attract*, settle*, establish*, occup*, breed*, population*, *introduce* , dispers*, capture*, trap*, "site selection", "habitat } \\
\text { selection", burrow, nest }\end{array}$ \\
\hline Additional terms for intervention & conspecific* ${ }^{*}$, heterospecific* social $^{*}$ \\
\hline Additional terms for outcome & conserv $^{*}$, manage $^{*}$ \\
\hline
\end{tabular}




\section{Bibliographic databases}

We will search multiple databases to retrieve various types of documents:

1. Web of Science-(https://webofknowledge.com).

i. Core collection.

ii. BIOSIS previews.

iii. Zoological record.

2. ProQuest-(https://www.proquest.com).

3. BioRxiv-(https://www.biorxiv.org).

We will use Web of Science to search three different databases to find studies within peer-reviewed published literature, conference abstracts, and book chapters. We will select the 'Topic' search field when performing our search in Web of Science. We will use ProQuest to find studies within Ph.D. dissertations and Master's theses, only searching on abstract or title. We will use BioRxiv to find studies within unpublished preprints. We will search the Animal Behavior and Cognition, Ecology, Evolutionary Biology, and Zoology collections in BioRxiv, and search on abstract or title. Search strings in BioRxiv cannot be longer than 128 characters so we will search on multiple smaller strings created from our original search string (eventually encompassing all search terms). All separate searches will be thoroughly documented in a spreadsheet as described below.

\section{Web-based search engines}

We will use Google Scholar to search the internet for relevant studies. Because Google Scholar Search is limited to one 'phrase' (enclosed in double quotation marks), one OR substring and 256 characters, we will adjust our search string accordingly by creating multiple search strings that fall within these parameters. All separate searches will be thoroughly documented in a spreadsheet as described below. The first 50 hits per search string, sorted by relevance, will be examined. If we find new journal articles that were not retrieved during other searches (e.g., using Web of Science) we will extend the use of Google Scholar to more than the first 50 hits (e.g., to the first 100 hits).

\section{Organizational/specialist websites}

We will search various organizational websites to retrieve additional publications and data within gray literature. We have compiled a list of 50 relevant websites in Additional file 2, many of which were recommended by stakeholders, including wildlife conservation managers and researchers from various international institutions.
Again, since the search options of each website might not be compatible with our search string, we may need to adjust searches accordingly. All search methods and results will be thoroughly documented in a spreadsheet as described below.

\section{Comprehensiveness of the search}

We used a benchmark list that included four studies (Additional file 1) to determine whether our search included well-known studies in the field. These studies were recommended to us by stakeholders.

All searchers will be documented thoroughly in a spreadsheet that will include: database name and URL, or website name and URL (for online search engines and gray literature), the exact search string used, the terms searched (i.e., topic or title), date and time of search, name of person performing the search, number of hits returned, and name and location of file containing the search results.

\section{Search update}

If the review takes more than 2 years to complete, we will redo our search and only include the years for which we lack data. All searches will be thoroughly documented in a spreadsheet as described above.

\section{Article screening and study eligibility criteria Screening process}

We will use CADIMA (systematic review software [21]) to store and organize search results. Duplicates will be identified and merged. The articles returned from searches will be screened on title and abstract simultaneously, and then on full text. Our main goal is to find studies that have used playbacks of heterospecific and/ or conspecific calls to attract wild animals for conservation purposes. Reviews will be kept, only to search for additional primary literature cited in the review. Conference abstracts will be assessed and if deemed relevant, the author(s) will be contacted to see if sharing of data is possible. We are only including studies available in English, and from all dates. Articles that are obtained, but not in English (or cannot be translated) will be listed in an appendix to the review. If we are uncertain of whether an article meets inclusion criteria, we will tend towards inclusion.

We will perform a consistency check to determine whether our inclusion criteria can be consistently applied to studies among different reviewers (e.g., low inter-individual variation). We will take a random selection of $10 \%$ of the total studies returned from our search and determine inter-reviewer agreement in study selection by calculating the Kappa value among three different reviewers. We will decide that our inclusion criteria 
are robust if the Kappa value is $>0.60$, which is generally deemed substantial agreement [22]. Before moving onto the full screening of studies, we will discuss reasons for inconsistencies among reviewers and attempt to resolve them (i.e., achieve high consistency in study selection). We will revise the criteria and redo the consistency check, by randomly selecting another $10 \%$ of total studies, if we receive a Kappa value $\leq 0.60$.

\section{Eligibility criteria}

Each study must include each of the criteria detailed in Table 3 (separated by PICO components).

All studies resulting from the study selection process should be experimental and contain either a BA (beforeafter), CI (comparator-intervention), or BACI (before/ after/comparator/intervention) type of design. We will exclude studies not conducted in the wild, expect for studies done in captivity if the study's outcomes meet one of our criterion (see Table 1). We will also exclude studies that use playbacks to understand aspects of animal communication (i.e., signal form, function, and evolution) or social relationships as these are not relevant to our primary question. The authors have not authored any articles that would be considered in this systematic review.
Blumstein has conducted many playback experiments, but neither of the authors have published empirical articles in this field related to wildlife management. Thus, we will be independent in decisions regarding inclusion and critical appraisal (see below) of studies. We will thoroughly document the articles excluded at full text with reasons for exclusion and provide this list as supplementary material in our review.

\section{Study validity assessment}

To determine differences in study validity and susceptibility to bias, various factors will be assessed to rank studies as having low, medium, or high susceptibility to bias (Table 4). We will record in detail how we assessed each study based on the factors in a transparent manner.

A BA design, in which the outcome is measured before and after the intervention is implemented, is susceptible to bias because temporal factors such as climate change can introduce confounding effects (in the case of a long-term BA study). This is especially a problem if the experiment is only conducted at a single site. Thus, CI and BACI designs, and multiple replicated sites, are the most valid and would be categorized as having low bias.

Table 3 Inclusion criteria

\begin{tabular}{ll}
\hline Component & Criteria \\
\hline Eligible populations & Animals of conservation concern, including both vertebrates and invertebrates \\
Eligible interventions & Acoustic playback(s) of conspecifics and/or heterospecifics (e.g., broadcast of bird song recording) \\
Eligible comparators & No playback (Cl) or to a time when no playback was used (BA) or both (BACl) \\
Eligible outcomes & Quantitative measurement of at least one of the following during the study attempt: success- \\
& ful capture of the target animals at the target location, occupation of the target animal at the \\
& target location, breeding of the target animal at the target location, establishment of nests, \\
& burrows, or territories by the target animal at the target location. For studies not related to the \\
& capture of animals, the outcome must include a target location that this different than where \\
& the target animal currently occupies and/or uses (for example, as temporary breeding ground)
\end{tabular}

Table 4 Critical appraisal criteria

\begin{tabular}{|c|c|c|c|}
\hline Factors under consideration & Low bias & Medium bias & High bias \\
\hline Study type & Experimental & & Observational \\
\hline Study design & $\mathrm{BACl}$ & $\mathrm{Cl}$ & BA \\
\hline Study plots & Homogeneous in size and habitat & $\begin{array}{l}\text { Homogeneous in size or } \\
\text { habitat }\end{array}$ & $\begin{array}{l}\text { Not homogeneous in } \\
\text { size or habitat }\end{array}$ \\
\hline Replication & $>3$ replicates & $2-3$ replicates & $<2$ replicates \\
\hline Duration of study ${ }^{a}$ & $>1$ year; multiple breeding seasons & $\begin{array}{l}<1 \text { year; longer than one } \\
\text { breeding season }\end{array}$ & One breeding season \\
\hline Account for confounding variables & Yes & Somewhat & No \\
\hline \multicolumn{4}{|l|}{ Methodology ${ }^{b}$} \\
\hline Statistical analyses ${ }^{b}$ & & & \\
\hline
\end{tabular}

\footnotetext{
${ }^{\text {a }}$ For studies in which the goal is long-term establishment or breeding and not capture of the animal
}

b These will be assessed individually based on each study's specific question and design 
Observational studies, if any are found, will be ranked as having a high susceptibility to bias.

The selection of study sites/plots could be important in affecting a study's susceptibility to bias. Ideally, plots should be relatively homogeneous in size, structure, and habitat so as not to introduce potential confounding variables. The duration of the study is also important. Playbacks used to attract animals can be targeted at animals making settlement decisions after dispersing from their natal territory, animals finding appropriate breeding grounds, or animals finding appropriate stopping locations during long-distance migration. The latter two involve sites that are usually only temporarily used. Ideally, studies will measure outcome(s) for more than 1 year to determine whether settlement or lack thereof persists in the long-term, which would ultimately be most useful for conservation managers. For studies that use playbacks to attract animals for capture, long-term behavioral responses are less important.

We will rank studies on susceptibly to bias using a validity assessment form (Table 4). The authors will make individual judgements on the validity of methodological details (sample sizes, details on playback(s) used and how they were implemented, number of visits, details on outcome measurement) and statistical analyzes depending on the type of study as these will vary based on research question and study design. Studies that have high susceptibility to bias in five or more factors will be excluded from our review. Any excluded studies will be listed in an appendix to the review, along with reasons for their exclusion. We will report each study's susceptibility to bias (high, medium, low) in our review. The critical appraisal may be used to qualify conclusions (i.e., weight studies in the synthesis) if we find large variance in biases among studies.

Following other systematic review protocols [23, 24], we will determine inter-reviewer consistency in critical appraisal by comparing meta-data entries for three separate reviewers for a randomly-selected subset of $10 \%$ of included studies. Any discrepancies among reviewers will be discussed and adjustments to the meta-data sheet and category codes will be adjusted until consensus is reached. Kappa values will not be calculated at this stage since our goal will be for total agreement on critical appraisal.

\section{Data coding and extraction strategy}

Meta-data will be extracted from studies that fulfill the selection criteria. Various types of data will be collected (see Additional file 3), including methodological information that could inform conservation managers on effective implementation of using playbacks to attract animals, and various covariates that could affect study outcomes (i.e., potential effect modifiers).

For data extraction, we will extract only quantitative study findings, including the mean(s), estimate(s) of variation (standard error, standard deviation, confidence interval, etc.), and sample size(s) of measured outcome(s). This information will be found in tables, figures, or in the text. We will use image analysis software (e.g., WebPlotDigitizer [25]) to extract means and measures of variation from figures when studies do not provide raw data or these values in text. If only raw data are presented, we will calculate the summary statistics. Authors will be contacted if these data are not clearly presented or extractable. Various data will be extracted, including details on the population and outcome components, and information on the study location, design, and methodology (see Additional file 3 for details).

If an article contains missing or unclear information or data, we will contact authors for clarifications. If authors do not respond, these studies may not be able to be included in the final review (in cases in which means and measures of variation are unknown) or they will be ranked lower during the critical appraisal process. We will record which studies we were unclear about, and include them in an appendix to the review, along with reasons for their exclusion. Extracted data records will be made available as additional files in the review.

To determine inter-reviewer consistency in data extraction, we will use the same methods as above during critical appraisal. We will compare meta-data and data entries for three separate reviewers for a randomly-selected subset of $10 \%$ of included studies. Any discrepancies among reviewers will be discussed and adjustments to the meta-data sheet and category codes will be adjusted until consensus is reached.

\section{Potential effect modifiers/reasons for heterogeneity}

We consulted with wildlife conservation managers (stakeholders) on potential sources of heterogeneity. We created list of factors that stakeholders identified as important or of potential importance (see below). We also include other effect modifiers that we presumed might influence the effectiveness of acoustic playbacks. Sources of heterogeneity are also presented in our list of meta-data to be extracted.

Effects related to study organism

- Taxa.

- Family.

- Guild.

- Species.

- Body size.

- Longevity.

- Nocturnal or diurnal. 
Effects related to location

- Country.

- Latitude and longitude.

- Habitat type.

Effects related to methodology

- Type of playback (conspecific, heterospecific, or both).

- Playbacks included at night (years/n).

- Playbacks directed at nocturnal migrants (years/n).

- Duration of study in a given year (in days).

- Duration of study in years.

- Duration of playback per day (in hours).

- Percent of time the stimulus is present during playback duration.

- Area (in ha) that playbacks are broadcast.

- Number of speakers used.

- Amplitude, frequency range, and volume of playback(s).

- Control auditory stimuli used (years/n).

- Stimulus of another sensory modality used (e.g., visual cue).

- Random silence incorporated into playback (years/n).

\section{Effects related to outcome}

- Type of outcome measured (e.g., playbacks might be more effective for short-term attraction of animals compared to long-term settlement).

\section{Data synthesis and presentation}

We will provide a narrative synthesis of data in our review that will synthesize the findings of all the studies. This will describe the body of evidence and how robust the overall findings are. Tables or graphical visualizations will be used to summarize these results, with the goal that we will be able to identify general themes or trends, consistency of the effect of the intervention, study clusters, patterns of factors or effect modifiers, and/or knowledge gaps.

Furthermore, we intend to include in our narrative synthesis whether we were able to address the questions from stakeholders. If not, we will highlight these important knowledge gaps. Stakeholders also suggested we create a roadmap for which questions remain unanswered and suggestions on how best to investigate them empirically. Finally, stakeholders expressed interest in potential negative effects of using playbacks. For instance, acoustic playbacks could successfully lure an animal to a desired habitat, but that animal might experience lower reproductive success within the habitat (see [26]). Such information, if found, would be included in our narrative synthesis. Once our review is complete, we will communicate our results back to the stakeholders whom we originally contacted.

If we find a sufficient amount of studies (more than two) that measure the same or similar outcome, we will perform a quantitative synthesis of the data (i.e., metaanalysis). In this case, studies will be weighted according to the amount of information they provide (e.g., by inverse variance). We will calculate Hedges' $g$ as a measure of effect size for each study, and combine these results to produce a single summary effect [27]. If we conduct a formal meta-analysis, we will be certain to calculate heterogeneity statistics and try to understand what sorts of species/life histories are associated with specific sorts of response. Where possible, we will estimate publication bias (i.e., statistically significant results are more likely to be published) and calculate fail-safe numbers, which estimate the amount of unpublished studies in the metaanalysis [28]. More specific details of quantitative analysis will only be known when articles have been assessed for their contents and quality.

\section{Additional files}

Additional file 1. List of benchmark studies used to create search string Additional file 2. List of grey literature sources to be used in the review. Additional file 3. List of data to be extracted from studies.

Authors' contributions

Both authors participated in the drafting and revision. Both authors read and approved the final manuscript approval of the manuscript.

\section{Author details}

${ }^{1}$ Department of Ecology and Evolutionary Biology, University of California, 621 Young Drive South, Los Angeles, CA 90095-1606, USA. ${ }^{2}$ Section of Herpetology, and Urban Nature Research Center, Natural History Museum of Los Angeles County, 900 Exposition Boulevard, Los Angeles, CA 90007, USA.

\section{Acknowledgements}

We thank Biljana Macura for instructing us at the October 2017 systematic review workshop at the Ben Gurion University of the Negev, Israel, and other participants of the workshop for their thoughtful comments on our review.

\section{Competing interests}

The authors declare that they have no competing interests.

Availability of data and materials

Not applicable.

Consent for publication

Not applicable.

Ethics approval and consent to participate

Not applicable. 


\section{Funding}

Both authors are currently supported by the National Science Foundation (DBI-1611562 to BJP; DEB-1557130 to DTB).

\section{Publisher's Note}

Springer Nature remains neutral with regard to jurisdictional claims in published maps and institutional affiliations.

Received: 28 August 2018 Accepted: 11 January 2019

Published online: 22 January 2019

\section{References}

1. Stamps JA. Conspecific attraction and aggregation in territorial species. Am Nat. 1988;131:329-47.

2. Swanson EM, Tekmen SM, Bee MA. Do female frogs exploit inadvertent social information to locate breeding aggregations? Can J Zool. 2007;85:921-32.

3. Andrews JE, Brawn JD, Ward MP. When to use social cues: conspecific attraction at newly created grasslands. Condor. 2015;117:297-305.

4. Ward MP, Schlossberg S. Conspecific attraction and the conservation of territorial songbirds. Conserv Biol. 2004;18:519-25.

5. Zanette LY, White AF, Allen MC, Clinchy M. Perceived predation risk reduces the number of offspring songbirds produce per year. Science. 2011;334:1398-401.

6. Madden N, Jehle R. Acoustic orientation in the great crested newt (Triturus cristatus). Amphibia-Reptilia. 2017;38:57-655.

7. Buxton RT, Jones IL. An experimental study of social attraction in two species of storm-petrel by acoustic and olfactory cues. Condor. 2012:114:733-43.

8. Fischer J, Noser R, Hammerschmidt K. Bioacoustic field research: a primer to acoustic analyses and playback experiments with primates. Am J Primatol. 2013;75:643-63.

9. Friesen MR, Beggs JR, Gaskett AC. Sensory-based conservation of seabirds: a review of management strategies and animal behaviours that facilitate success. Biol Rev. 2017;92:1769-84.

10. Ahlering MA, Arlt D, Betts MG, Fletcher RJ Jr, Nocera JJ, Ward MP. Research needs and recommendations for the use of conspecificattraction methods in the conservation of migratory songbirds. Condor 2010;112:252-64.

11. Hettena AM, Munoz N, Blumstein DT. Prey responses to predator's sounds: a review and empirical study. Ethology. 2014;120:427-52.

12. Kress SW. The use of decoys, sound recordings, and gull control for re establishing a tern colony in Maine. Colon Waterbirds. 1983;6:185-96.
13. Ahlering MA, Faaborg J. Avian habitat management meets conspecific attraction: If you build it, will they come? Auk. 2006;123:301-12.

14. Hahn BA, Silverman ED. Social cues facilitate habitat selection: American redstarts establish breeding territories in response to song. Biol Lett. 2006;2:337-40.

15. Anich NM, Ward MP. Using audio playback to expand the geographic breeding range of an endangered species. Divers Distrib. 2017;23:1499-508.

16. Sih A, Ferrari MCO, Harris DJ. Evolution and behavioural responses to human-induced rapid environmental change. Evol Appl. 2011;4:367-87.

17. Tuomainen U, Candolin U. Behavioural responses to human-induced environmental change. Biol Rev. 2011:86:640-57.

18. Berger-Tal O, Greggor AL, Macura B, Adams CA, Blumenthal A, Bouskila A, et al. Systematic reviews and maps as tools for applying behavioral ecology to management and policy. Behav Ecol. 2018. https://doi. org/10.1093/beheco/ary130.

19. Greggor AL, Berger-Tal O, Blumstein DT, Angeloni L, Bessa-Gomes C, Blackwell BF, et al. Research priorities from animal behaviour for maximising conservation progress. Trends Ecol Evol. 2016;31:953-64.

20. Collaboration for Environmental Evidence (2018) Guidelines and Standards for Evidence Synthesis in Environmental Management. Version 5.0. AS Pullin, GK Frampton, B Livoreil, G Petrokofsky, Eds. https://www.envir onmentalevidence.org/information-for-authors. Accessed 16 Nov 2018

21. CADIMA (2018) https://www.cadima.info/. Accessed 8 Nov 2018

22. McHugh ML. Interrater reliability: the kappa statistic. Biochem Med. 2012;22:276-82.

23. Ward-Campbell BMS, Valere B. What are the impacts of small-scale dredging activities on inland fisheries productivity? A systematic review protocol. Environ Evid. 2018;7:1-7.

24. Land M, Haddaway NR, Hedlund K, Jørgensen HB, Kätterer T, Isberg PE. How do selected crop rotations affect soil organic carbon in boreo-temperate systems? A systematic review protocol. Environ Evid. 2017;6:1-8.

25. Rohatgi A (2018) WebPlotDigitizer. https://automeris.io/WebPlotDigitizer. Accessed 11 Nov 2018

26. Grendelmeier A, Arlettaz R, Olano-Marin J, Pasinelli G. Experimentally provided conspecific cues boost bird territory density but not breeding performance. Behav Ecol. 2017;28:174-85.

27. Lajeunesse MJ. Facilitating systematic reviews, data extraction and meta-analysis with the metagear package for R. Methods Ecol Evol. 2016;7:323-30

28. Fragkos KC, Tsagris M, Frangos CC. Exploring the distribution for the estimator of Rosenthal's 'fail-safe' number of unpublished studies in metaanalysis. Commun Stat-Theory Methods. 2017;46:5672-84.
Ready to submit your research? Choose BMC and benefit from:

- fast, convenient online submission

- thorough peer review by experienced researchers in your field

- rapid publication on acceptance

- support for research data, including large and complex data types

- gold Open Access which fosters wider collaboration and increased citations

- maximum visibility for your research: over $100 \mathrm{M}$ website views per year

At BMC, research is always in progress.

Learn more biomedcentral.com/submissions 